\title{
Numerical Simulation of the Coupled Turbulent Flow and Macroscopic Solidification in Continuous Casting with Electromagnetic Brake
}

\author{
Deok-Soo KIM, Woo-Seung $\mathrm{KIM}^{11}$ and Kee-Hyeon $\mathrm{CHO}^{2)}$ \\ Graduate school of Mechanical Engineering, Hanyang University, Seongdong-ku, Seoul 133-791 Korea. \\ 1) Department of Mechanical Engineering, Hanyang University, Ansan, Kyunggi-do 425-791 Korea. \\ 2) Research Institute of Industrial Science \& Technology (RIST), Pohang, Kyung-buk 790-600 Korea.
}

(Received on October 4, 1999; accepted in final form on March 7, 2000)

\begin{abstract}
A computer program has been developed for analyzing the three-dimensional, steady conservation equations for transport phenomena in a slab continuous casting process with Electromagnetic Brake (EMBr) to investigate the effect of $\mathrm{EMBr}$ on the turbulent melt-flow, temperature fields, and macroscopic solidification of the molten metal. The enthalpy-porosity relation was employed to suppress the velocity within a mushy region. A revised low-Reynolds number $k-\varepsilon$ turbulence model was used to consider the turbulent effects. The electromagnetic field was described by Maxwell equations. The application of $\mathrm{EMBr}$ to the mold region results in the decrease of the transfer of superheat to the narrow face, the increase of temperature in freesurface region and most part of the melt of submold region, and the higher temperature gradients near the solidifying shell. The increasing magnetic flux density has effect mainly on the surface temperature of the solidifying shell at the narrow face, hardly on that at the wide face. It is seen that in the presence of EMBr, a thicker solidifying shell is obtained at the narrow face of slab.
\end{abstract}

KEY WORDS: numerical simulation; turbulent flow; macroscopic solidification; electromagnetic brake $(\mathrm{EMBr})$; continuous casting $(\mathrm{CC})$ process.

\section{Introduction}

Electromagnetic Brake (EMBr) is an useful tool to control and redistribute the molten metal flows in a mold region using a transverse static magnetic field applied to the mold of continuous caster. The practical effect of EMBr on the melt-flow phenomena is regarded as the reduction of two major recirculating flows which are due to the melt jet flow supplied into caster through a submerged entry nozzle (SEN). A suppression of the recirculating flows results in the elimination or reduction of cast defects which relates closely to the melt-flow in the mold region, such as the entrapment of inclusions and mold powder, longitudinal and transverse cracks of cast, static and dynamic waves at a meniscus, and remelting at the narrow sides of strand, etc. ${ }^{1,2)}$ Thereby, the control of melt-flow in the mold region is essential to eliminate or reduce the melt-flow-related defects, and to result in an increase of the productivity of steel in a continuous casting (CC) process.

The CC process is related with the liquid-to-solid phasechange. Furthermore, the turbulent flow and heat transfer in the melt affects the growth of solidifying shell. However, most work, carried out on the CC process with an EMBr system, has been limited to the analysis of the EMBr-applied melt flow with no consideration of solidification. Thus, the correlation between flow pattern and solidified shell in the CC process has not been clearly established. ${ }^{3-6)}$ Although a minority of study ${ }^{7,8)}$ considered the solidification phenomena, the effects of EMBr on the temperature field and solidified shell in CC process were not shown in those works. Therefore, it may be meaningful that the investigation of the controled flow pattern by EMBr and solidification is performed.

It is difficult owing to the facility scale and cost consumption, to obtain the desired experimental data for an industrial scale of CC process. Thus, a majority of previous work, mainly depending on numerical simulations, has been done on the basis of ultimately limited conditions. ${ }^{9-13)}$ In particular, two-dimensional analysis has been performed to reduce the computational cost for the analysis of the coupled turbulent flow and solidification. Although the assumption of two-dimensional modeling can be permitted for a round billet caster, the two-dimensional modeling of the square billet or rectangular slab casters has some limitations. Especially, for the slab caster with small aspect ratio (thickness/width) of slab, the cooling effect in the slabthickness direction can not be practically taken into account through the two-dimensional model. Therefore, for more realistic modeling of the slab casting process, the three-dimensional analysis of the coupled momentum, solidification, and induced electric field is required to be carried out. $^{14)}$ 


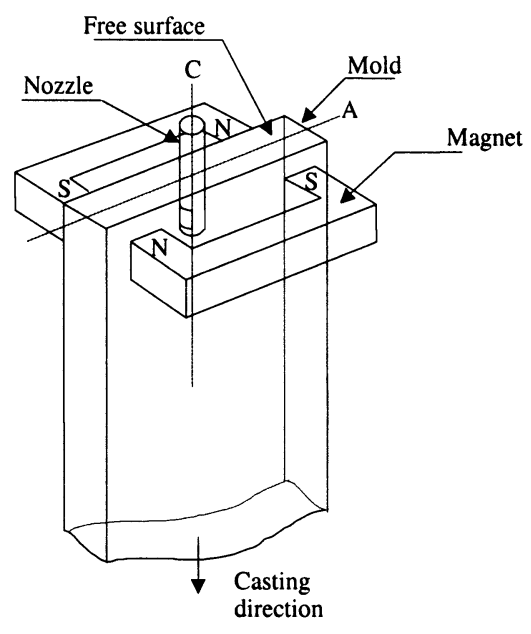

(a)

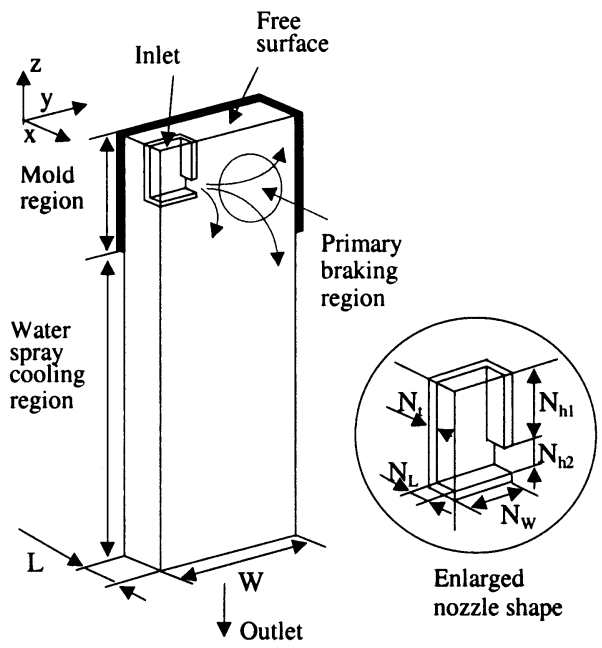

(b)

Fig. 1. Schematic of a typical caster with EMBr and its computational domain. (a) Casting mold (b) Computational domain.

In the present study, a three-dimensional analysis of the coupled turbulent flow, solidification, and induced electric field in a slab CC process with EMBr system, is performed using a developed computer program. And based on the corresponding results, the velocity and temperature distributions are investigated. The suppression effects of velocity in phase-change zone are considered depending on a porosity-enthalpy relationship. The turbulent characteristics of melt-flow in the liquid region are taken into account using a revised version of low-Reynolds number $k-\varepsilon$ turbulence model by Launder and Sharma. ${ }^{15)}$

\section{Theory}

In a commercial continuous caster, the melt is fed through a submerged nozzle from tundish into a vibrating, water-cooled mold. The jet-flow of melt is suppressed by an electromagnetic force due to the EMBr system applied to the mold. The melt from which sufficient heat is extracted by the mold, forms a solidified shell, and moves down into secondary cooling zones by water spray. This study is interested in the vertical part only of the continuous caster, composed of the vertical and curved parts. A schematic diagram of slab caster with EMBr system is shown in Fig. 1(a). The computational domain shown in Fig. 1(b) is chosen based on the symmetry of caster shape displayed in Fig. 1(a). A circular shape of nozzle is assumed to be the rectangular cross-sectional flow-area with an equivalent hydraulic diameter.

\subsection{Mathematical Model}

In a commercial scale of CC process, the melt-flow fed into the caster through a bifurcated SEN has the characteristics of turbulent flow. Standard $k-\varepsilon$ model of turbulence is especially applicable to the flow regions with high turbulent Reynolds number $\mathrm{Re}_{\mathrm{t}}=\rho k^{2} / \mu \varepsilon$ and cannot be applied near the solid walls, where the viscous effects become dominant. Therefore, the use of either wall function approach or some form of damping factor is essential to consider the laminar sublayer. In the CC process, solidification profile is un- known a priori and has to be determined as a part of solution. Thus, it is very difficult to employ the wall functions. In this study, a revised version of low-Reynolds number $k-\varepsilon$ turbulence model by Launder and Sharma, ${ }^{15)}$ adopted by a few previous studies, ${ }^{13,14)}$ is used to consider the turbulent characteristics. A treatment for the release of latent heat in a mushy zone depends on a single-domain-based enthalpy method. The separation approach to a total enthalpy into the sensible enthalpy $(h)$ and latent heat $(\Delta H)$ is adopted for energy conservation equation. The following assumptions are introduced in this study;

1. The CC process is at steady state, and the melt fed into the caster behaves as an incompressible Newtonian fluid.

2. The free-surface covered with mold powder and slag is thermally insulated, and is maintained at a constant level.

3. The density and thermophysical properties for the solid and liquid metals are invariant and equal.

4. The liquid fraction in mushy region varies linearly with temperature variation.

5. The velocity of solid phase is equal to the casting speed.

6. The electromagnetic characteristics of melt are uniform and isotropic.

Depending on the above postulations, time-averaged governing equations considered in this study take the following form :

Continuity equation

$$
\frac{\partial U_{i}}{\partial x_{i}}=0
$$

Momentum equation

$$
\begin{aligned}
& \frac{\partial\left(\rho U_{j} U_{i}\right)}{\partial x_{j}}=-\frac{\partial P}{\partial x_{i}}+\frac{\partial}{\partial x_{j}}\left[\left(\mu+\mu_{\mathrm{t}}\right) \frac{\partial U_{i}}{\partial x_{j}}\right] \\
& +\frac{\partial}{\partial x_{j}}\left(\mu_{\mathrm{t}} \frac{\partial U_{j}}{\partial x_{i}}\right)-\frac{2}{3} \rho \frac{\partial k}{\partial x_{i}} \delta_{i j}+F_{\mathrm{m}, i}-\frac{\mu}{K_{\mathrm{p}}}\left(U_{i}-U_{i, \mathrm{~s}}\right)
\end{aligned}
$$


Energy equation

$$
\frac{\partial\left(\rho U_{j} h\right)}{\partial x_{j}}=\frac{\partial}{\partial x_{j}}\left[\left(\frac{\mu}{\operatorname{Pr}}+\frac{\mu_{\mathrm{t}}}{\sigma_{\mathrm{t}}}\right) \frac{\partial h}{\partial x_{j}}\right]-\frac{\partial\left(\rho U_{j} \Delta H\right)}{\partial x_{j}}
$$

Turbulent kinetic energy equation

$$
\frac{\partial\left(\rho U_{j} k\right)}{\partial x_{j}}=\frac{\partial}{\partial x_{j}}\left[\left(\mu+\frac{\mu_{\mathrm{t}}}{\sigma_{\mathrm{k}}}\right) \frac{\partial k}{\partial x_{j}}\right]+P_{\mathrm{t}}-\rho \varepsilon-D_{k}
$$

Dissipation rate of turbulent kinetic energy

$$
\begin{aligned}
\frac{\partial\left(\rho U_{j} \varepsilon\right)}{\partial x_{j}}= & \frac{\partial}{\partial x_{j}}\left[\left(\mu+\frac{\mu_{\mathrm{t}}}{\sigma_{\varepsilon}}\right) \frac{\partial \varepsilon}{\partial x_{j}}\right] \\
& +C_{\varepsilon 1} f_{1} P_{\mathrm{t}} \frac{\varepsilon}{k}-C_{\varepsilon 2} f_{2} \rho \frac{\varepsilon^{2}}{k}+E_{\varepsilon}
\end{aligned}
$$

where

$$
\begin{aligned}
& \mu_{\mathrm{t}}=C_{\mu} f_{\mu} \rho \frac{k^{2}}{\varepsilon} \\
& P_{\mathrm{t}}=\mu_{\mathrm{t}}\left(\frac{\partial U_{i}}{\partial x_{j}}+\frac{\partial U_{j}}{\partial x_{i}}\right) \frac{\partial U_{i}}{\partial x_{j}} \\
& D_{k}=2 \mu \frac{\partial \sqrt{k}}{\partial x_{i}} \frac{\partial \sqrt{k}}{\partial x_{i}} \\
& E_{\varepsilon}=\frac{2 \mu \mu_{\mathrm{t}}}{\rho} \frac{\partial^{2} U_{i}}{\partial x_{j} \partial x_{k}} \frac{\partial^{2} U_{i}}{\partial x_{j} \partial x_{k}} \\
& F_{\mathrm{m}, i}=J_{j} B_{k}-J_{k} B_{j} \\
& \operatorname{Re}_{\mathrm{t}}=\frac{\rho k^{2}}{\mu \varepsilon}
\end{aligned}
$$

$$
\begin{gathered}
f_{\mu}=\exp \left(-3.4 /\left(1+\mathrm{Re}_{\mathrm{t}} / 50\right)^{2}\right), \quad f_{1}=1, \\
f_{2}=1-0.3 \exp \left(-\mathrm{Re}_{\mathrm{t}}^{2}\right), \quad C_{\varepsilon 1}=1.44, \quad C_{\varepsilon 2}=1.92, \\
C_{\mu}=0.09, \quad \sigma_{k}=1.0, \quad \sigma_{\varepsilon}=1.3, \quad \sigma_{\mathrm{t}}=0.9 \ldots \ldots .
\end{gathered}
$$

The local latent heat $\Delta H$ in Eq. (3) is a function of liquid fraction, and can be expressed as in Eq. (7):

$$
\Delta H=\left\{\begin{array}{ll}
\Delta H_{\mathrm{f}}, & T>T_{\text {liq }} \\
f_{\mathrm{l}} \Delta H_{\mathrm{f}}, & T_{\mathrm{sol}}<T<T_{\text {liq }} \\
0, & T<T_{\mathrm{sol}}
\end{array}\right\}
$$

where $f_{1}$ represents the local liquid fraction. $T_{\text {sol }}$ and $T_{\text {liq }}$ are the solidus and liquidus temperatures, respectively. $\Delta H_{\mathrm{f}}$ is the latent heat of fusion. The last term of right hand side in momentum Eq. (2) is added as the damping source term due to Darcy law for a porous media, depending on an enthalpy-porosity relation, to consider the velocity suppression for a mushy region. ${ }^{16)}$ Here, a permeability $K_{\mathrm{p}}$ can be expressed, in the form of a function of local liquid fraction, depending on Kozeny-Carman equation.

$$
K_{\mathrm{p}}=\frac{f_{1}^{3}+q}{D_{0}\left(1-f_{1}\right)^{2}}
$$

where $D_{0}$ is a constant, related to the morphology of porous

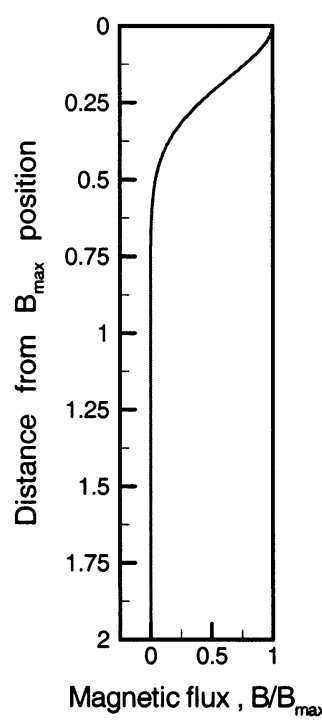

Fig. 2. Magnetic flux density used for simulation.

media. ${ }^{17)}$ The value of $D_{0}$ is estimated from the expression $D_{0}=180 / d^{2}$, which was employed by some previous literature. $^{13,14)}$ The value of $d$ is of the order of $1 \times 10^{-4} \mathrm{~m}$. The constant $D_{0}$ is assumed to be $1.8 \times 10^{10}$ in this study. The constant $q$, introduced to avoid the division by zero, is set at 0.001 . Similar source terms are introduced for $k$ and $\varepsilon$ equations, to force the values of $k$ and $\varepsilon$ in solid zone be zero.

\subsection{Induced Braking Force}

During electrically conducting melt motion under static magnetic field perpendicular to the direction of melt stream, the braking force is induced, which acts in the opposite direction to the melt stream, resulting in suppression of the melt flow. The braking force per unit volume of melt, is calculated by Eq. (9).

$$
\vec{F}_{\mathrm{m}}=\vec{J} \times \vec{B}
$$

where $\vec{F}_{\mathrm{m}}$ represents the induced braking force, $\vec{J}$ is the induced current density, and $\vec{B}$ is the magnetic flux density. When the current density is known for an externally applied static magnetic field, the braking force can be estimated. The magnetic flux density distribution used in this study, based on Ref. 5), is shown Fig. 2, assuming that the static magnetic field has maximum value in the center of magnetic field $(y=0.44 \mathrm{~m}, z=-0.41 \mathrm{~m})$, and decreases with distance from the center. The current density is calculated by Maxwell equations ${ }^{18)}$ under the assumption that the induced magnetic field is negligible in comparison with an externally applied magnetic field.

$$
\begin{gathered}
\nabla \cdot \vec{J}=0 \ldots \ldots \ldots \\
\vec{J}=\sigma\left(\vec{E}+\vec{V}_{1} \times \vec{B}\right) \\
\vec{E}=-\nabla \phi \ldots \ldots
\end{gathered}
$$

where $\vec{V}_{1}$ represents the liquid velocity, $\sigma$ is the electrical conductivity, and $\vec{E}$ is the electric field intensity. From the above Eqs. (10)-(12), The following Poisson equation of the electrical potential $\phi$ is obtained:

$$
\nabla \cdot(\sigma \nabla \phi)=\nabla \cdot \sigma\left(\vec{V}_{1} \times \vec{B}\right)
$$

Once $\phi$ is estimated by Eq. (13), the braking force is calcu- 
lated by Eqs. (9) and (11).

\subsection{Boundary Conditions}

For the computational domain of caster shown in Fig. 1(b), boundary conditions used for this numerical simulation are as follows.

Nozzle-Inlet: The values of the dependent variables, except for the current density, are assumed to be constant at the nozzle-inlet, and the normal gradient of current density vanishes. The values of $k$ and $\varepsilon$ are based on the following semi-empirical expressions suggested by Lai et al., ${ }^{19)}$ and used in subsequent literature ${ }^{14)}$ :

$$
\begin{aligned}
& U=V=0, \quad W=W_{\text {in }}, \quad h=h_{\text {in }}, \quad \frac{\partial J_{\mathrm{z}}}{\partial z}=0, \\
& k=0.01 W_{\text {in }}^{2}, \quad \varepsilon=C_{\mu} k^{1.5} / 0.05 D \ldots \ldots \ldots \ldots \ldots \ldots \ldots \ldots \ldots \ldots \ldots . . .
\end{aligned}
$$

where $W_{\text {in }}$ is the inlet velocity, and $D$ is the hydraulic diameter of nozzle-inlet.

Symmetry Faces: The velocity and current density components normal to the symmetry faces are zero, and there is no existence of the normal gradients of other dependent variables. Hence :

$$
\begin{gathered}
\frac{\partial V}{\partial x}=\frac{\partial W}{\partial x}=\frac{\partial h}{\partial x}=\frac{\partial k}{\partial x}=\frac{\partial \varepsilon}{\partial x}=U=J_{x}=0 \quad \text { at } x=L \\
\frac{\partial U}{\partial y}=\frac{\partial W}{\partial y}=\frac{\partial h}{\partial y}=\frac{\partial k}{\partial y}=\frac{\partial \varepsilon}{\partial y}=V=J_{y}=0 \quad \text { at } y=0
\end{gathered}
$$

Free-Surface: The same boundary conditions as those used for the symmetry faces are utilized at the free-surface. The surface tension effect is not taken into account on the basis of the previous literature. ${ }^{13,14)}$.

$$
\frac{\partial U}{\partial z}=\frac{\partial V}{\partial z}=\frac{\partial h}{\partial z}=\frac{\partial k}{\partial z}=\frac{\partial \varepsilon}{\partial z}=W=J_{z}=0 \quad \text { at } z=0
$$

Outlet: The outlet is selected at the position of $3 \mathrm{~m}$ apart from the free-surface. The fully developed flow conditions are considered at the outlet. All of the dependent variables and the current density have the zero axial gradients :

$$
\frac{\partial U}{\partial z}=\frac{\partial V}{\partial z}=\frac{\partial W}{\partial z}=\frac{\partial h}{\partial z}=\frac{\partial k}{\partial z}=\frac{\partial \varepsilon}{\partial z}=\frac{\partial J_{z}}{\partial z}=0
$$

Moving Wall: The solidified shell, moving with casting speed, is cooled down in sequence, by the mold and water spray. The heat removal by the mold and water spray is treated using the average heat transfer coefficient $(\gamma)$ between the solid surface and the surrounding. Hence :

$$
\begin{aligned}
& W=W_{\mathrm{c}}, \quad U=V=k=\varepsilon=0, \quad J_{x}=J_{y}=0, \\
& \frac{\partial h}{\partial x}=\frac{\partial h}{\partial y}=-\frac{\gamma}{K}\left(h_{\mathrm{s}}-h_{\mathrm{a}}\right) \ldots \ldots \ldots \ldots \ldots \ldots \ldots \ldots \ldots \ldots \ldots \ldots \ldots \ldots \ldots
\end{aligned}
$$

where $W_{\mathrm{c}}$ is the casting speed. $K$ is the thermal conductivity of the melt, $h_{\mathrm{s}}$ and $h_{\mathrm{a}}$ and correspond to the solid surface and surrounding enthalpies, respectively. The values of average heat transfer coefficients chosen for this numerical study, are $1500 \mathrm{~W} / \mathrm{m}^{2} /{ }^{\circ} \mathrm{C}$ and $750 \mathrm{~W} / \mathrm{m}^{2} /{ }^{\circ} \mathrm{C}$, respectively, for the mold and water spray cooling regions. ${ }^{14)}$

\subsection{Numerical Analysis}

The governing Eqs. (1)-(5) and (10) were discretized using a control-volume-based finite difference approach. The discretization of convective terms depended on the hybrid scheme, and SIMPLER algorithm of Patankar ${ }^{20)}$ was used as the numerical solution technique.

The solutions to the discretization equations with the prescribed boundary conditions, are obtained iteratively employing the line-by-line TDMA solver. The following convergence criteria was adopted in the present simulation:

$$
\left|\frac{R_{i}^{m+1}-R_{i}^{m}}{R_{i}^{m}}\right| \leq \varepsilon
$$

where superscripts $m+1$ and $m$ represent iterative computation steps, and subscript $i$ is nodal point at which the values of dependent variables are calculated. $R$ is residual and the constant $\varepsilon$ is set at $10^{-5}$. The under-relaxation factors during computation were set to be 0.4 and 0.25 for velocity and scalar quantities, respectively. The non-uniform grid system of $18 \times 27 \times 31$, based on the previous work, ${ }^{14)}$ was adopted in the computation. The numerical procedure can be summarized as follows :

(1) The braking force is calculated by equations related to electromagnetic field.

(2) Velocity and pressure are obtained by SIMPLER algorithm.

(3) Temperature is predicted by energy equation.

(4) The procedures (1)-(3) are repeated until the converged solutions are obtained.

\section{Results and Discussion}

The investigation of the coupled turbulent flow and solidification in the upper vertical part of slab continuous caster with $\mathrm{EMBr}$ system, was carried out in the present study. The inlet velocity for casting speed of $-0.015 \mathrm{~m} / \mathrm{s}$, was calculated by using the mass balance between the inlet and outlet. The superheat of melt is $26^{\circ} \mathrm{C}$. The thermophysical properties are listed in Table 1. The details for the enlarged nozzle shape shown in Fig. 1(b) are as follows; $N_{\mathrm{t}}=$ $0.0336 \mathrm{~m}, N_{\mathrm{L}}=0.029 \mathrm{~m}, N_{\mathrm{W}}=0.098 \mathrm{~m}, N_{\mathrm{h} 1}=0.29 \mathrm{~m}$, and $N_{\mathrm{h} 2}=0.1 \mathrm{~m}$.

The rough features of the flow pattern and temperature distribution in a slab CC process without an EMBr system are shown in Figs. 3 and 4. Figure 3(a) represents velocity vectors, and Fig. 3(b) shows the solidus- and liquidus lines. As can be observed in Fig. 3(a), the jet flow of melt leaving from nozzle-exit impinges on the narrow side of mold, and is divided into two oppositely directed flows, resulting in the formation of the strongly recirculating flows in the upper and lower regions, respectively. Figure 3(b) shows that the mushy zone forms around the vertical centrally symmetric face parallel to the narrow face in the outlet region. This results from the thickness-directional cooling effect in the slab CC process.

The solidus and liquidus lines at various transverse cross-sectional planes downstream from the top free-sur- 
Table 1. Thermophysical properties and parameters used for simulation.

\begin{tabular}{l|l|l|l}
\hline Parameters & Values & Parameters & Values \\
\hline Cast size $(2 \mathrm{~W} \times 2 \mathrm{~L})$ & $1.32 \mathrm{~m} \times 0.2 \mathrm{~m}$ & Mold length & $0.70 \mathrm{~m}$ \\
\hline $\begin{array}{l}\text { Heat transfer coefficient } \\
\text { in mold region }\end{array}$ & $1500 \mathrm{~W} / \mathrm{m}^{2} / \mathrm{C}$ & Viscosity & $6.2 \times 10^{-3} \mathrm{~kg} / \mathrm{m} / \mathrm{s}$ \\
\hline $\begin{array}{l}\text { Heat transfer coefficient in } \\
\text { water spray cooling region }\end{array}$ & $750 \mathrm{~W} / \mathrm{m}^{2} /{ }^{\circ} \mathrm{C}$ & Latent heat of fusion & $270 \mathrm{~kJ} / \mathrm{kg}$ \\
\hline Thermal conductivity & $31.0 \mathrm{~W} / \mathrm{m} /{ }^{\circ} \mathrm{C}$ & Liquidus temperature & $1454^{\circ} \mathrm{C}$ \\
\hline Specific heat & $700 \mathrm{~J} / \mathrm{kg} /{ }^{\circ} \mathrm{C}$ & Solidus temperature & $1400^{\circ} \mathrm{C}$ \\
\hline Density & $7000 \mathrm{~kg} / \mathrm{m}^{3}$ & Electrical conductivity & $7.14 \times 10^{5} / \Omega \mathrm{m}$ \\
\hline
\end{tabular}

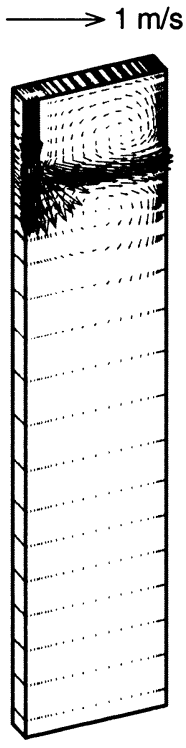

(a)

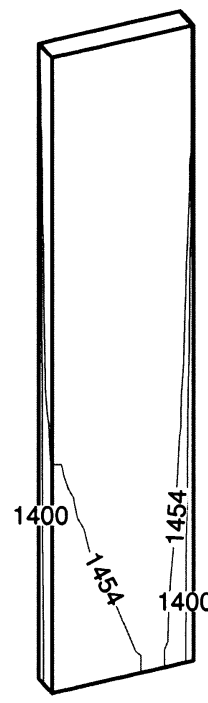

(b)
Fig. 3. Three-dimensional surface plot for a casting speed of $-0.015 \mathrm{~m} / \mathrm{s}$ and superheat of $26^{\circ} \mathrm{C}$. (a) velocity vectors (b) liquidus and solidus isotherms.

face are displayed in Fig. 4. The thin solidified shell is seen to form along the wide and narrow faces in the upper mold. The shell thickness as well as the mushy zone progressively increase during moving downstream, as more heat is extracted through the solidified shell by the water-cooled mold. In the corner region appear the round shapes of the shell layer and mushy zone, owing to the higher heat-extraction rate by both sides of the mold. In the narrow face around $z=-0.381 \mathrm{~m}$, the shell thickness remains nearly unchanged, and the very thin mushy region forms, because the melt supplied into the caster impinges on this region, and forms the flow toward the wide face of mold. The mushy region, observed in the center region of slab cross-sectional plane at $z=-2.096 \mathrm{~m}$, is due to the cooling effect at the wide face.

Although not given as a figure in this study, the good agreements, only except for some deviation in the mushy zone shape near the nozzle-exit, were verified from the comparison of Figs. 3 and 4 with the previous work ${ }^{14)}$ that investigated a three-dimensional turbulent flow and solidification for Fig 1(b), In the work ${ }^{14)}$ was not given the suppression approach to the velocity, kinetic energy $k$, and dissipation rate of kinetic energy $\varepsilon$ in the mushy region, which strongly affects the mushy shape. The deviation from the previous work ${ }^{14)}$ is considered to originate mainly from the uncertainty of suppression approach to the melt-flow and
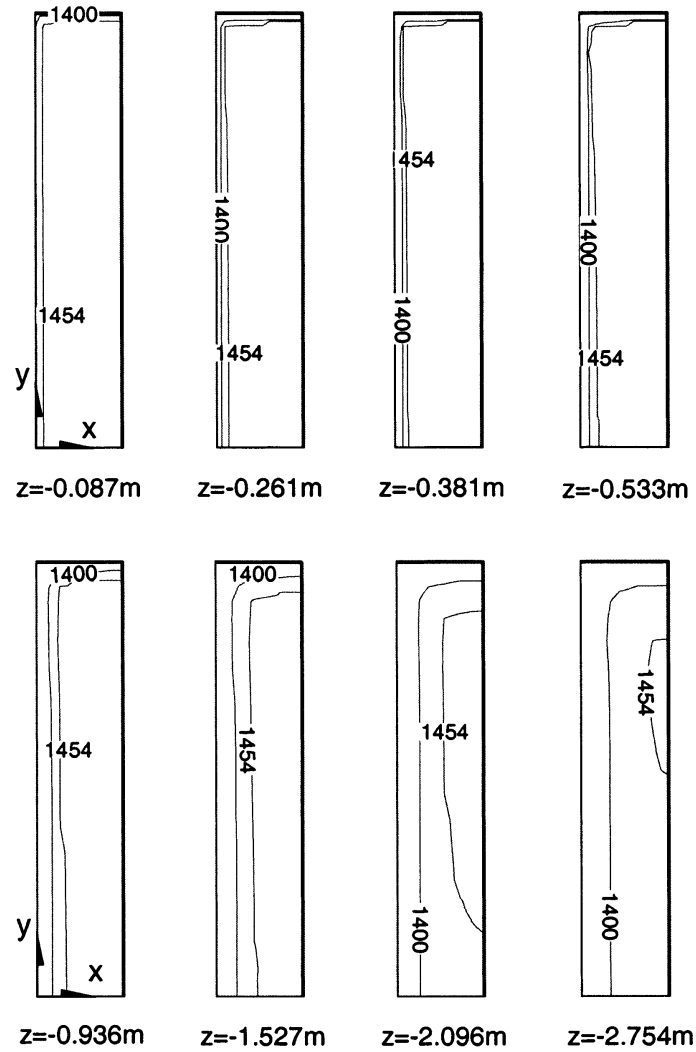

Fig. 4. Contours of solidus and liquidus temperatures at various transverse cross-sectional planes ( $x-y$ planes).

turbulent effect in the mushy zone.

The influences of the variation of magnetic flux density on the flow pattern are shown in Fig. 5. It is noted that no back flow appears at the outlet boundary for the various magnetic flux densities. As can be seen in Fig. 5(a), in case of no EMBr system the velocity vectors in the upper region of caster show strong flow stream. Figure 5(b) shows that the EMBr system causes the effectual suppression of meltflow, and results in the reduction of velocity in the mold region and the more uniform melt-flow below the mold as well. However, as observed in Fig. 5(c), the excessive magnetic flux density causes the melt-flow in the mold region, not being suppressed sufficiently within the primary region of magnetic field, to flow toward the top free-surface, and to move down along the narrow face of mold. ${ }^{6}$ ) Therefore, it can be known that too large EMBr-strength gives rise to the improper flow streams in the upper caster.

The temperature fields at the vertical centrally symmetric plane $(x=L)$ paralleled to the wide face and at the free-surface, for various magnetic flux densities, are displayed in Figs. 6 and 7, respectively. The suppression of jet melt-flow 


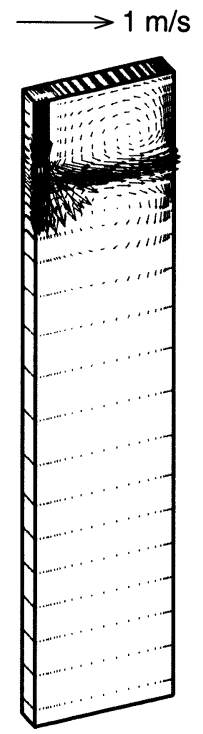

(a) $B_{\max }=0.0$

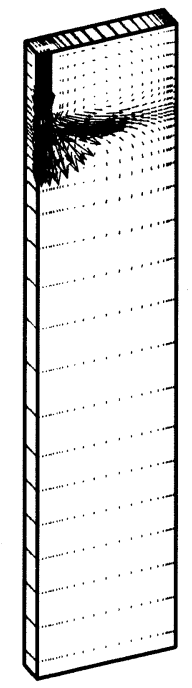

(b) $B_{\text {max }}=0.13$

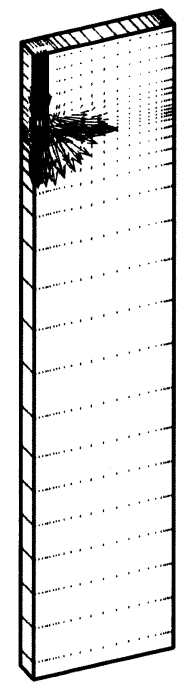

(c) $B_{\max }=0.25$
Fig. 5. Three-dimensional surface plots of velocity vectors for various magnetic flux densities.

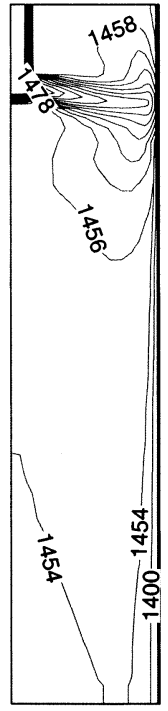

(a) $B_{\max }=0.0$

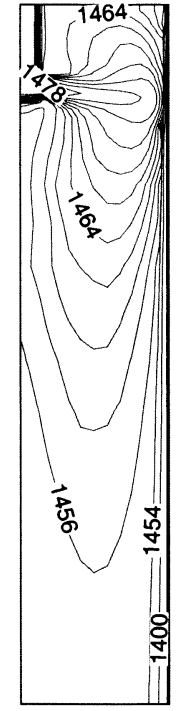

(b) $B_{\text {max }}=0.13$

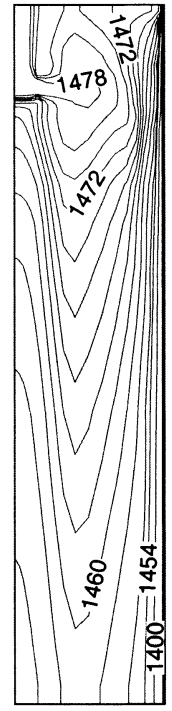

(c) $B_{\max }=0.25$
Fig. 6. Temperature fields at the vertical centrally symmetric plane paralleled to the wide face for various magnetic flux densities.

by $\mathrm{EMBr}$ system causes the reduction of advective heat transfer to the narrow face. Thereby, the superheat is not removed sufficiently at the narrow face of mold, and is transported to the free-surface and lower regions, resulting in the increase of energy level in the overall liquid region, and the increase in temperature gradients near the solidified shell. And observing the isotherm lines in the lower region, the Vshapes of isotherm lines appear by increasing the magnetic flux density. Besides, in Figs. 6(b) and 6(c) diminishes the mushy zone to form in the lower-left region, which can be seen in Fig. 6(a).

From observing Fig. 7, the temperature rise at the overall free-surface takes place with the increased magnetic flux density. The free-surface zone should retain properly high temperature to melt the mold powder for a stable lubrica- (a) $B_{\max }=0.0$

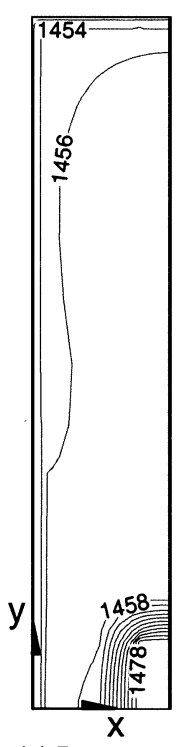

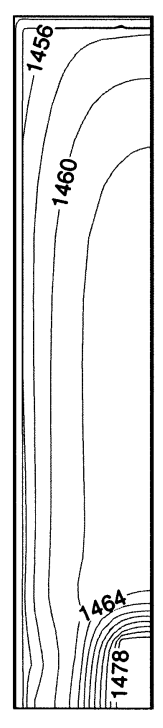

(b) $B_{\max }=0.13$

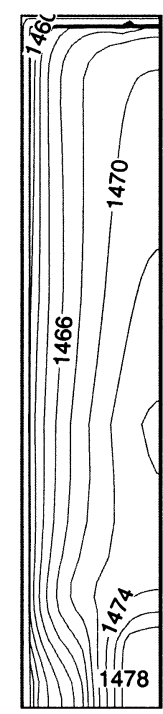

(c) $B_{\max }=0.25$
Fig. 7. Temperature fields at the free surface level for various magnetic flux densities.

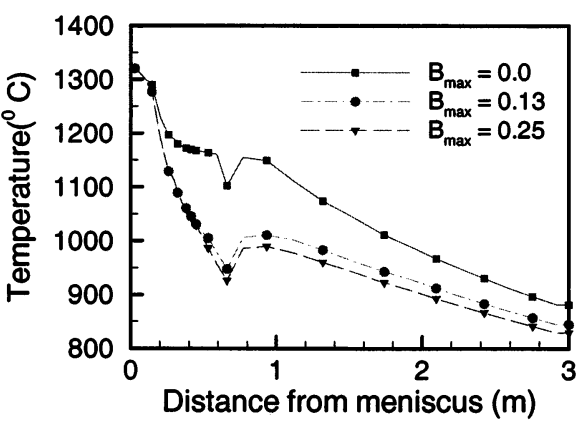

(a)

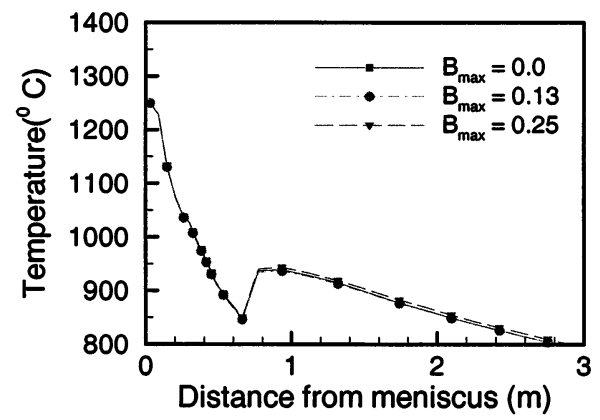

(b)

Fig. 8. Surface temperature at vertical centerline of solidifying shell. (a) narrow face (b) wide face.

tion during CC process. Thereby, in relation to the dependence of free-surface temperature on the superheat, an increase in free-surface temperature by applying EMBr system, may help melt-superheat to be reduced, and to increase the melting performance of mold powder, compared to that for no EMBr system.

The effects of the EMBr on the surface temperature of solidified shell are shown in Fig. 8. Figures 8(a) and 8(b) show the temperature profiles along the wide and narrow faces, respectively. As can be seen in Fig. 8, while an increase in magnetic flux density causes the decrease in shellsurface temperature at the narrow face, and hardly affects 


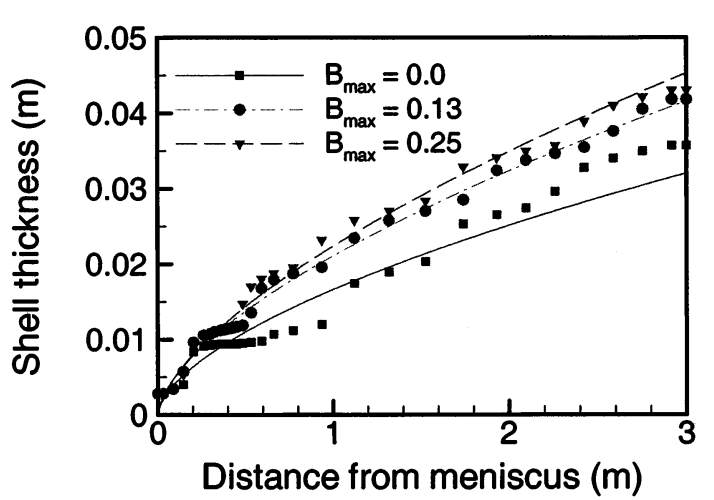

Fig. 9. Solidifying shell thicknesses at narrow face for various magnetic flux densities.

the surface temperature at the wide face. The EMBr system leads mainly to a decrease in narrow face temperature. The reheating effect at the wide face is larger by $40 \%$ than that at the narrow face.

The influences of the melt-flow controlled by EMBr system on the growth of solidifying shell are shown in Fig. 9. Symbols represent the data calculated from the simulation, and solid lines are curve-fitted to the corresponding values. The shell growth scarcely appears around the distance $0.4 \mathrm{~m}$ downstream from the free-surface. This is due to the fact that the impingement of the melt through a nozzle on the narrow face limits the shell growth. In addition, it is seen in Fig. 9 that an increase in magnetic flux density causes the thicker solidified shell. The reason for this is that, by increasing EMBr effect, the convective heat transfer, which obstructs the shell growth, between the melt and solidifying shell decreases, and the increased temperature gradients near the solidifying shell increases the diffusion heat flux to shell surface.

\section{Conclusions}

In the present study a three-dimensional analysis of the coupled turbulent flow and solidification was performed in the slab continuous casting process with $\mathrm{EMBr}$ system. And based on the corresponding results, the velocity and temperature distributions were investigated. The following conclusions can be summarized :

(1) In case of no EMBr system, two strongly recirculating flows appear in the upper and lower regions of the caster, respectively. And the mushy zone appears, due to the cooling effect at the wide face, in the center region of slab cross-sectional plane around the downstream caster. However, the EMBr applied to the mold causes the effectual suppression of melt-flow, and results in the reduction of velocity in the mold region and the more uniform melt-flow below the mold as well.

(2) The application of EMBr to the mold region results in the decrease of the transfer of superheat to the narrow face, the increase of temperature in the free-surface and most liquid of submold regions, and the higher temperature gradients near the solidifying shell.
(3) In relation to the dependence of free-surface temperature on the superheat, an increase in free-surface temperature by applying EMBr system, may help melt-superheat to be reduced, and to increase the melting performance of mold powder, compared to that for no EMBr system. The V-shapes of isotherm lines appear in the lower region, by increasing the magnetic flux density.

(4) While an increase in magnetic flux density causes a decrease in shell-surface temperature at the narrow face, and hardly affects the surface temperature at the wide face. The reheating effect at the wide face is larger by $40 \%$ than that at the narrow face.

(5) In the presence of EMBr, there decreases the convective heat transfer, which obstructs the shell growth, between the melt and solidifying shell, and the increased temperature gradients near the solidifying shell increases the diffusion heat flux to the shell surface, resulting in the thicker solidified shell at the narrow face.

\section{REFERENCES}

1) H. Hackl, S. G. Kollberg and G. Tallbäck: 1st European Conf. on Continuous Casting, ABB Metallurgy AB, Västeras, (1991), 2401.

2) S. G. Kollberg, H. R. Hackl and P. J. Hanley: Iron Steel Eng., 73 (1996), No. 7, 24.

3) Y. Yoneyama, E. Takeuchi, K. Matsuzawa, I. Sawada, Y. Hattori and Y. Kishida: Nippon Steel Technical Report, No. 45, Apr., (1990), 30.

4) H. Tozawa, H. Kitaoka, K. Sorimachi, H. Ishizuka, M. Ohnishi and S. Kakihara: Proc. of 6th Int. Iron and Steel Cong., ISIJ, Tokyo (1990), 438.

5) A. Idogawa, M. Sugizawa, S. Takeuchi, K. Sorimachi and T. Fujii: Mater. Sci. Eng., A173 (1993), 293.

6) Y.-S. Hwang, P.-R. Cha, H.-S. Nam, K.-H. Moon and J.-K. Yoon: ISIJ Int., 37 (1997), 659.

7) K. Takatani, K. Nakai, N. Kasai, T. Watanabe and H. Nakajima: Proc. of 6th Int. Iron and Steel Cong., ISIJ, Tokyo, (1990), 430.

8) A. F. Lehman, G. R. Tallbäck, S. G. Kollberg and H. R. Hackl: Int. Symp. on Electromagnetic Processing of Materials, ISIJ, Tokyo, (1994), 372.

9) X. Huang, B. G. Thomas and F. M. Najjar: Metall. Trans. B, 23B, (1992), 339.

10) S. K. Choudhary and D. Mazumdar: ISIJ Int., 34 (1994), 584.

11) W. Shyy, Y. Pang, G. B. Hunter, D. Y. Wei and M. H. Chen: Int. J. Heat Mass Transfer, 35, No. 5, (1992), 1229.

12) T. G. O'Connor and J. A. Dantzig: Metall. Mater. Trans. B, 25B (1994), 443.

13) M. R. Aboutalebi, M. Hasan and R. I. L. Guthrie: Numer. Heat Transfer A, 28 (1995), 279.

14) S. H. Seyedein and M. Hasan: Int. J. Heat Mass Transfer, 40 (1997), No. $18,4405$.

15) B. E. Launder and B. I. Sharma: Lett. Heat Mass Transfer, 1 (1974), 131.

16) V. R. Voller and C. Prakash: Int. J. Heat Mass Transfer, 30 (1987), No. 8,170

17) D. R. Poirier: Metall. Trans. B, 18B (1987), 245.

18) W. F. Hughes and F. J. Young: The Electromagnetodynamics of Fluids, John Wiley \& Sons, Inc., New York (London) Sydney, (1996), 140.

19) K. Y. M. Lai, M. Salcudean, S. Tanaka and R. I. L. Guthrie: Metall. Trans. B, 17B (1986), 449.

20) V. G. Patankar: Numerical Heat Transfer and Fluid Flow, Hemisphere, New York, (1980).

21) B. Lally, L. T. Biegler and H. Henein: Metall. Trans. B, 22B (1991), 641. 